\title{
Preoperative Predictors of Early Mortality Risk in People with Osteosarcoma of the Extremities Treated with Standard Therapy
}

\author{
Ke Tian, Peng-ju Li, Yan Zhang \\ Department of Orthopaedic Surgery, The First Affiliated Hospital of Zhengzhou University, Zhengzhou, 450052, Henan, People's Republic of China
}

Correspondence: Yan Zhang, Department of Orthopaedic Surgery, The First Affiliated Hospital of Zhengzhou University, No. I East Jianshe Road, District of Erqi, Zhengzhou, 450052, Henan, People's Republic of China, Tel +86-13783610679, Email zhangyan74II0I@I63.com

Purpose: More precise identification of osteosarcoma patients with high early death risk and enhanced early follow-up of these patients, such as increasing the frequency of postoperative chest computed tomography (CT) and local magnetic resonance imaging (MRI) examinations, may improve the overall survival of patients. The primary purpose of this research is to explore the risk factors related to early mortality in patients with osteosarcoma under standard treatment.

Patients and Methods: Our research included 87 osteosarcoma patients who had undergone standard treatment and had a Karnofsky (KPS) $\geq 70$. We define patients who die within 2 years of diagnosis as early death. The clinical characteristics and laboratory indicators of patients with osteosarcoma were collected and analyzed retrospectively.

Results: The median follow-up time was 32 months (4-91 months). Early deaths occurred in 13 patients. Early death of patients with osteosarcoma is related to tumor metastasis $(\mathrm{P}<0.001)$, tumor size $>5 \mathrm{~cm}(\mathrm{P}=0.049)$, high-level neutrophil-lymphocyte ratio (NLR) $(\mathrm{P}=0.035)$, high-level fibrinogen (FIB) $(\mathrm{P}=0.038)$, and higher $\mathrm{D}$-dimer $(\mathrm{DD})(\mathrm{P}=0.025)$. According to our results of multivariate Cox analysis, tumor metastasis status at diagnosis $(\mathrm{P}<0.001)$, NLR $(\mathrm{P}=0.039)$ and FIB $(\mathrm{P}=0.023)$ are independent risk factors in predicting early mortality in osteosarcoma patients. The "Osteosarcoma Early Mortality Nomogram" has a C index of 0.871 , and the calibration curve performs best compared with the ideal model in predicting mortality in 1 year.

Conclusion: Tumor metastasis status, NLR, and FIB are independent risk factors in predicting early mortality in osteosarcoma patients. The early follow-up of patients with tumor metastasis, high NLR, and high FIB should be strengthened.

Keywords: osteosarcoma, early death, biomarker, survival

\section{Introduction}

Osteosarcoma is a high-grade primary malignant bone sarcoma that predominantly affects children and youth. ${ }^{1}$ Studies have reported that the 5-year overall survival of osteosarcoma patients is about $60-70 \%$ when given the standard treatment of surgery combined with chemotherapy. ${ }^{2}$ Unfortunately, the development of new drugs and other treatments has reached a plateau, and the prognosis of patients with osteosarcoma has not improved substantially since the 1970s. ${ }^{3,4}$ Clinical characteristics can be used to identify high-risk patients and formulate corresponding treatment plans. However, patients with similar clinical features often have completely different prognoses, even after receiving the same standard treatment. More accurate identification of high-risk patients and greater individualization of treatment plans may help to improve prognoses. Recent research has therefore focused on the development of biomarkers that can more exactly distinguish patients at high risk of death. Recently, several potential biomarkers have been developed, such as circulating tumor DNA (ctDNA), non-coding RNA (NcRNA), circulating tumor cell (CTC) ${ }^{5-7}$ However, owing to the cost of testing and strict technical requirements of biopsy, these markers have not yet been applied in a clinical setting. There is thus an urgent need for fast, convenient, accurate and cost-effective tools for preoperative risk assessment to develop personalized treatment plans. 
Early death of patients with cancer is related to many factors, ${ }^{8,9}$ including operation failure or poor clinical condition resulting in an inability to complete the entire treatment plan; There is no doubt that such early deaths are predictable However, early deaths have also occurred in some individuals with osteosarcoma who have a good level of physical fitness and who received successful standardized treatment; early identification of these patients could enable enhanced follow-up and supportive treatments that may yield net clinical benefits. Any potential markers for use in their identification should be easily available and cost-effective.

Recent research have shown that preoperative hematological markers such as neutrophil-lymphocyte ratio (NLR) and platelet-lymphocyte ratio (PLR) can reflect the tumor microenvironment of patients and can be used to distinguish highrisk patients. ${ }^{10,11}$ Markers such as NLR, PLR, and lactate dehydrogenase (LDH) have been shown to have prognostic value in a variety of cancer types, including osteosarcoma. ${ }^{12,13}$

To date, little research has explored factors affecting early death in people with osteosarcoma who receive standard treatment for this disease. Therefore, the main purpose of this study was to explore preoperative risk factors related to early death among people with osteosarcoma who received standard treatment.

\section{Patients and Methods}

\section{Patients Included and Excluded from the Study}

Our research retrospectively analyzed data from people with osteosarcoma recruited in the First Affiliated Hospital of Zhengzhou University diagnosed from January 2014 to October 2020. The inclusion criteria were as follows: 1) patients with osteosarcoma confirmed by histopathology; 2) patients with tumors located in the extremities; 3) patients with good general health and treatment tolerance (preoperative and postoperative Karnofsky performance status (KPS) scores $\geq$ 70); 4) patients with medical records and complete laboratory test results available for collection; 5) patients who received a full course of standard treatment consisting of preoperative neoadjuvant chemotherapy, surgery, postoperative adjuvant chemotherapy or surgery and postoperative adjuvant chemotherapy. Exclusion criteria were as follows: 1) patients who did not receive treatment in the First Affiliated Hospital of Zhengzhou University or who failed to complete a complete treatment plan; 2) patients with poor general health and/or treatment tolerance (preoperative and postoperative KPS scores $<70$ ); 3) patients with tumors are located in the non-extremities; 4) Patients with infections, hematopathy, or autoimmune diseases that may affect laboratory test results before treatment; 5) patients with severe postoperative complications (such as pulmonary embolism).

Regular follow-ups were carried out for each patient, with a final follow-up in May 2021. The research protocol for this study was approved by the Institutional Review Board and Medical Ethics Committee of the First Affiliated Hospital of Zhengzhou University in accordance with the Declaration of Helsinki. Since this study was retrospective and the data were all anonymized, informed consent was waived.

\section{Data Collection and Processing}

The main purpose of our research is to explore the factors related to the patient's early death. Previous research has explored such factors in cancer patients. ${ }^{14}$ In these studies, early mortality is defined as death within 1 year of receiving treatment. ${ }^{15,16}$ In our study, we defined early death as the death that occurred in the first $30 \%$ of the follow-up time (2 years). The following data were collected from hospital case files associated with each individual included in our study: patient's age, gender, KPS score, tumor metastasis status, tumor size, and laboratory test results. Preoperative hematologic markers included: alkaline phosphatase (ALP), NLR, PLR, lymphocyte-to-monocyte ratio (LMR), red blood cell distribution width (RDW), prognostic nutritional index (PNI; defined as albumin $(\mathrm{g} / \mathrm{L})+0.005 \times$ total lymphocyte count/ $\mathrm{mm}^{3}$ ), activated partial thromboplastin time (APTT), prothrombin time (PT), fibrinogen levels (FIB), and D-dimer levels (DD). Patients with KPS scores $\geq 70$ were considered to be in good health. ${ }^{17}$ Overall survival (OS) was the main endpoint of our research, defined as the interval between the date of tumor resection and the date of death of the patient or the date of last follow-up, whichever was earlier. 


\section{Statistical Analysis}

Independent samples $\mathrm{T}$ tests were used to analyze differences in continuous variables, and chi-squared tests were used to assess differences in categorical variables. Time-dependent receiver operating characteristic curves (TROC) were used to calculate the optimal cutoff value for each hematologic marker. TROC was conducted using the survivalROC package in R. According to the calculated cut-off value, patients with osteosarcoma are divided into high hematological maker level group and low hematological maker level group. Subsequently, we used univariate Cox analysis to determine which hematologic markers and clinical variables were associated with early death in people with osteosarcoma. Clinical variables and hematologic markers that were significant in our univariate Cox regression analyses were included in further multivariate Cox regression analyses. Hazard ratios (HRs) and P values for significant clinical variables and hematologic indicators were presented in forest plots. Kaplan-Meier analyses were used to generate survival curves, and Log rank tests were used to compare the survival rates of individuals in different risk groups. Finally, a nomogram to enable prediction of the risk of early death in people with osteosarcoma was constructed based on clinical variables and hematologic markers with significant associations with early death in the univariate Cox regression analysis. The predictive capacity and accuracy of the constructed nomogram were evaluated using the $\mathrm{C}$ index and calibration curve, respectively. Decision curve analysis was used to evaluate whether the nomogram could be clinically beneficial. All $\mathrm{T}$ tests and chi-square tests were performed in SPSS (version 21.0), and all other analyses were performed in $\mathrm{R}$ (version 4.0.5). The $\mathrm{R}$ code involved in our statistical analysis can be found in the Supplementary Information. $\mathrm{P}<$ 0.05 was used as the threshold for statistical significance throughout this study.

\section{Results}

\section{Characteristics of Patients with Osteosarcoma and Cut-Off Values of Hematological Markers}

We initially identified 128 osteosarcoma patients who were diagnosed and treated surgically at the First Affiliated Hospital of Zhengzhou University. Of these, 87 patients were included in the study based on our inclusion and exclusion criteria.

Table 1 shows the details of the individuals with osteosarcoma included in our research. As shown in Table 1, the median follow-up time for all patients was 32 (range, 4-91) months. The median age of patients was 17 years, and the median ALP value was 150. Median NLR: 1.92 (1.58-2.65), PLR: 125.9 (96.7-168.4), LMR: 4.04 (3.05-5.2), RDW: 12.7 (12.3-13.4), PNI: 52.5 (48.5-57.2), PT: 10.5 (10.0-11.2), APTT: 33.1 (30.9-35.4), FIB: 3.17 (2.73-3.52), DD: 0.16 (0.092-0.73). Cutoff values for the nine hematologic factors were calculated by TROC; these are shown in Supplementary Table 1 and Supplementary Figure 1 (NLR: 2.529, PLR: 142.174, LMR: 7.667, RDW: 13.40, PNI: 57.60, PT: 10, APTT: 39.10, FIB: 3.180, DD: 0.560). People with osteosarcoma were stratified into two groups based on the cutoff values. Factors related to early mortality were compared in patients with osteosarcoma. As shown in Table 2, early mortality was found to be associated with metastatic status, NLR, FIB levels, and DD levels. Early death appeared to be associated non-significantly with tumor size, PLR, and PT levels.

\section{Risk Factors for Early Mortality in Patients with Osteosarcoma}

As shown in Figure 1A, the results of our univariate Cox analysis indicated that tumor metastasis $(\mathrm{P}<0.001)$, tumor volume $>5 \mathrm{~cm}(\mathrm{P}=0.049)$, high NLR $(\mathrm{P}=0.035)$, high FIB level $(\mathrm{P}=0.038)$, and high DD level $(\mathrm{P}=0.025)$ were all significantly associated with early death. Subsequently, the area under the ROC curve (AUC) was calculated to evaluate the predictive power of these five variables. As shown in Figure 2, tumor metastatic status had the most significant predictive power, followed by NLR, then DD (AUC $=0.819,0.713$ and 0.669 , respectively). Finally, the results of our multivariate Cox analysis indicated that tumor metastatic status (hazard ratio $[\mathrm{HR}]=9.806,95 \%$ confidence interval $[\mathrm{CI}]$ 2.813-34.180, $\mathrm{P}<0.001$ ), NLR (HR $=3.648,95 \%$ CI 1.067-12.468, $\mathrm{P}=0.039)$ and FIB (HR $=4.746,95 \%$ CI $1.244-$ $18.113, \mathrm{P}=0.023$ ) were independent predictors of early mortality in people with osteosarcoma (Figure 1B).

We then focused on the two hematologic markers NLR and FIB and conducted survival analyses and Kaplan-Meier survival curves for these factors, which showed that the OS of people osteosarcoma in the high NLR group (> 2.529) was 
Table I Patient Demographics and Clinical Characteristics of Extremities Osteosarcoma Who Received Standard Treatment

\begin{tabular}{|l|c|}
\hline Characteristics & Patients, N = 87 \\
\hline Age, years, median (IQR) & $17(9-67)$ \\
Gender, n (\%) & $53(60.9)$ \\
Male & $34(39.1)$ \\
Female & \\
Metastasis status, n (\%) & $69(79.3)$ \\
YES & $18(20.7)$ \\
NO & \\
Size & $60(68.9)$ \\
$<5$ & $27(31.1)$ \\
>5 & $150(43-900)$ \\
ALP, median (IQR) & $1.92(1.58-2.65)$ \\
NLR, median (IQR) & $125.9(96.7-168.4)$ \\
PLR, median (IQR) & $4.04(3.05-5.2)$ \\
LMR, median (IQR) & $12.7(12.3-13.4)$ \\
RDW, median (IQR) & $52.5(48.5-57.2)$ \\
PNI, median (IQR) & $10.5(10.0-11.2)$ \\
PT, median (IQR) & $33.1(30.9-35.4)$ \\
APTT, median (IQR) & $3.17(2.73-3.52)$ \\
FIB, median (IQR) & $0.16(0.092-0.73)$ \\
DD, median (IQR) & $2.72(0.31-7.58)$ \\
Overall survival, years, median, range & \\
\hline
\end{tabular}

lower than those in the low NLR group $(\leq 2.529, \mathrm{P}=0.005$, Figure $3 \mathrm{~A})$. In addition, the survival curve also revealed that low FIB values were associated with better OS than high FIB values $(\mathrm{P}=0.026$, Figure 3B).

\section{Construction of Osteosarcoma Early Mortality Nomogram}

We constructed an Osteosarcoma Early Mortality Nomogram based on the five variables that were found to be significantly associated with early death in our univariate Cox regression. Each variable included in the nomogram was assigned a score in our multivariate Cox analysis. The sum of the scores for these variables gave the total score for the nomogram, which corresponds to the risk of early death. As shown in Figure 4A, compared with other variables, tumor metastasis at diagnosis had the highest score (100 points). The Osteosarcoma Early Mortality Nomogram had a $\mathrm{C}$-index of 0.871 . The calibration curve showed that the nomogram had the best fit with the ideal model when predicting death within 1 year (Figure 4B). The results of our DCA show that a combined model brings higher net clinical benefits compared with the clinical characteristic model (Figure 4C).

\section{Discussion}

Since 1970, the OS of people with osteosarcoma has plateaued. Newly-diagnosed individuals typically have a five-year survival rate of approximately $60-70 \%$ after receiving standard treatment with surgery and chemotherapy. ${ }^{18}$ The factors affecting poor prognosis in some patients are obvious, including failure to receive a full course of standardized treatment. However, some patients in good physical condition have surprisingly poor prognoses after completing the standard treatment plan. Therefore, this study focused on people with osteosarcoma who had KPS scores $\geq 70$ and who received standard treatment to explore the factors related to early mortality in this population. Many recent studies have shown that hematologic biomarkers, which are measured from peripheral blood samples, can distinguish people with a high risk of death among those with tumors. In contrast with genetic molecular biomarkers, the measurement of preoperative hematologic markers is cheap, non-invasive, and relatively easy. Therefore, in this study, we collected and analyzed clinical data and hematology test results for 87 people with osteosarcoma who met the inclusion criteria. Our results 
Table 2 Comparison of Preoperative Risk Factors for Early Death in Extremities Osteosarcoma Patients Received Standard Treatment

\begin{tabular}{|c|c|c|c|}
\hline Characteristics & $\begin{array}{c}\text { Death Within } 2 \\
\text { Years (13) }\end{array}$ & $\begin{array}{c}\text { Death After } 2 \\
\text { Years (74) }\end{array}$ & P-value \\
\hline \multicolumn{4}{|l|}{ Age, years } \\
\hline Median & 19 & 16 & 0.247 \\
\hline Range & $10-23$ & $9-67$ & \\
\hline \multicolumn{4}{|l|}{ Gender } \\
\hline Male & 10 & 43 & 0.202 \\
\hline Female & 3 & 31 & \\
\hline \multicolumn{4}{|c|}{ Metastasis status, n (\%) } \\
\hline YES & 7 & 11 & 0.001 \\
\hline No & 6 & 63 & \\
\hline \multicolumn{4}{|l|}{ Size, cm, n (\%) } \\
\hline$\leq 5 \mathrm{~cm}$ & 6 & 54 & 0.055 \\
\hline$>5 \mathrm{~cm}$ & 7 & 20 & \\
\hline \multicolumn{4}{|l|}{ ALP } \\
\hline Median & 180 & 147 & 0.439 \\
\hline Range & $69-408$ & $43-900$ & \\
\hline \multicolumn{4}{|l|}{ NLR, n (\%) } \\
\hline$\leq 2.529$ & 6 & 56 & 0.031 \\
\hline$>2.529$ & 7 & 18 & \\
\hline \multicolumn{4}{|l|}{ PLR, n (\%) } \\
\hline$\leq 142.174$ & 6 & 52 & 0.091 \\
\hline$>142.174$ & 7 & 22 & \\
\hline \multicolumn{4}{|l|}{ LMR, n (\%) } \\
\hline$\leq 7.667$ & 12 & 72 & 0.366 \\
\hline$>7.667$ & I & 2 & \\
\hline \multicolumn{4}{|l|}{ RDW, n (\%) } \\
\hline$\leq 13.40$ & 9 & 59 & 0.401 \\
\hline$>13.40$ & 4 & 15 & \\
\hline \multicolumn{4}{|l|}{ PNI, n (\%) } \\
\hline$\leq \mathbf{5 7 . 6 0}$ & 10 & 59 & 0.819 \\
\hline$>57.60$ & 3 & 15 & \\
\hline \multicolumn{4}{|l|}{ PT, n (\%) } \\
\hline$\leq 10.0$ & I & 23 & 0.084 \\
\hline$>10.0$ & 12 & 51 & \\
\hline \multicolumn{4}{|l|}{ APTT, n (\%) } \\
\hline$\leq 39.10$ & 10 & 65 & 0.295 \\
\hline$>39.10$ & 3 & 9 & \\
\hline \multicolumn{4}{|l|}{ FIB, n (\%) } \\
\hline$\leq 3.180$ & 3 & 42 & 0.022 \\
\hline$>3.180$ & 10 & 32 & \\
\hline \multicolumn{4}{|l|}{ DD, n (\%) } \\
\hline$\leq 0.560$ & 6 & 58 & 0.016 \\
\hline$>0.560$ & 7 & 16 & \\
\hline
\end{tabular}

indicate that preoperative tumor metastatic status, NLR, and FIB levels are associated with early death among people with osteosarcoma. Subsequently, we constructed an Osteosarcoma Early Mortality Nomogram incorporating clinical variables and hematologic indicators. The Osteosarcoma Early Mortality Nomogram had a C index of 0.871 and performed best compared to the ideal model when predicting mortality within 1 year; the constructed nomogram had satisfactory predictive performance. We recommend that this should be used to identify those who are at risk of high 


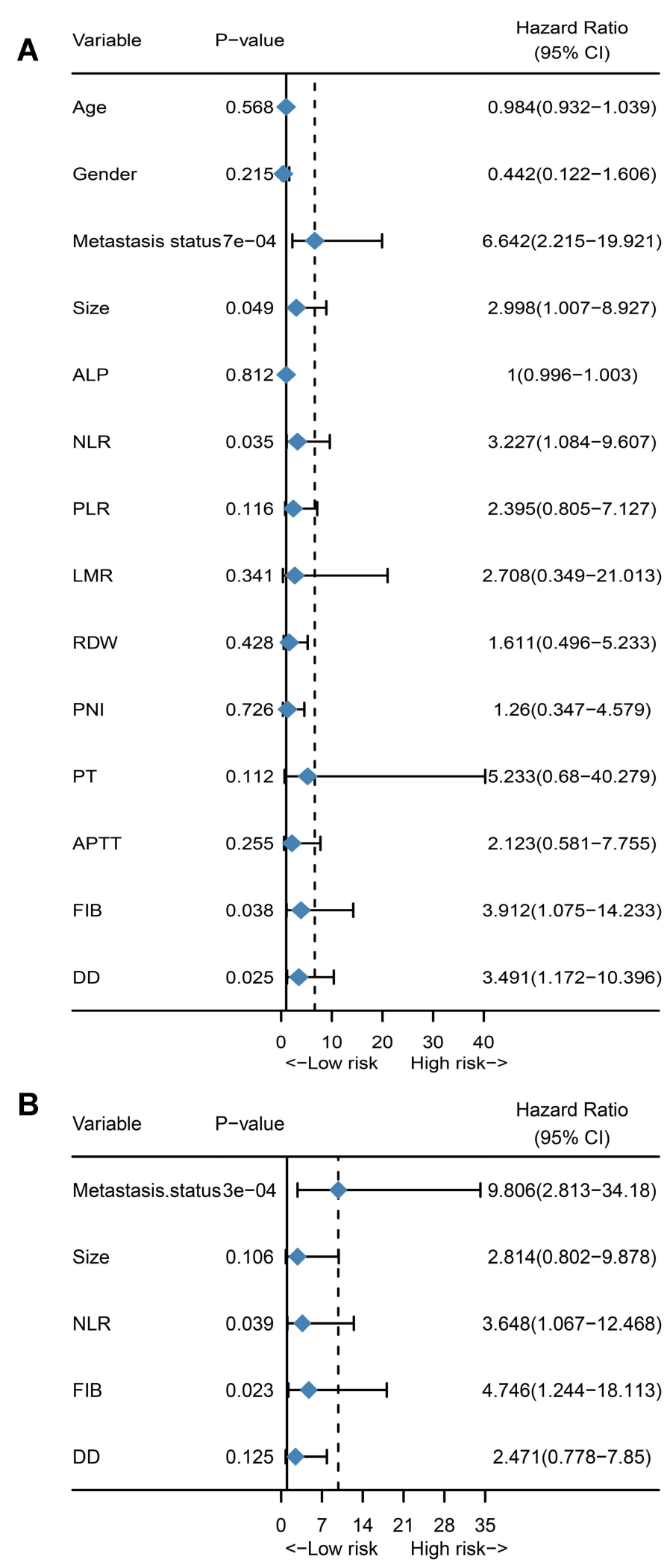

Figure I Evaluation of independent risk factors for early death in osteosarcoma patients. (A) Univariate Cox regression results for hematology markers and clinical features of patients with osteosarcoma. (B) Multivariate Cox regression results for variables that have significant significance in univariate Cox analysis. 


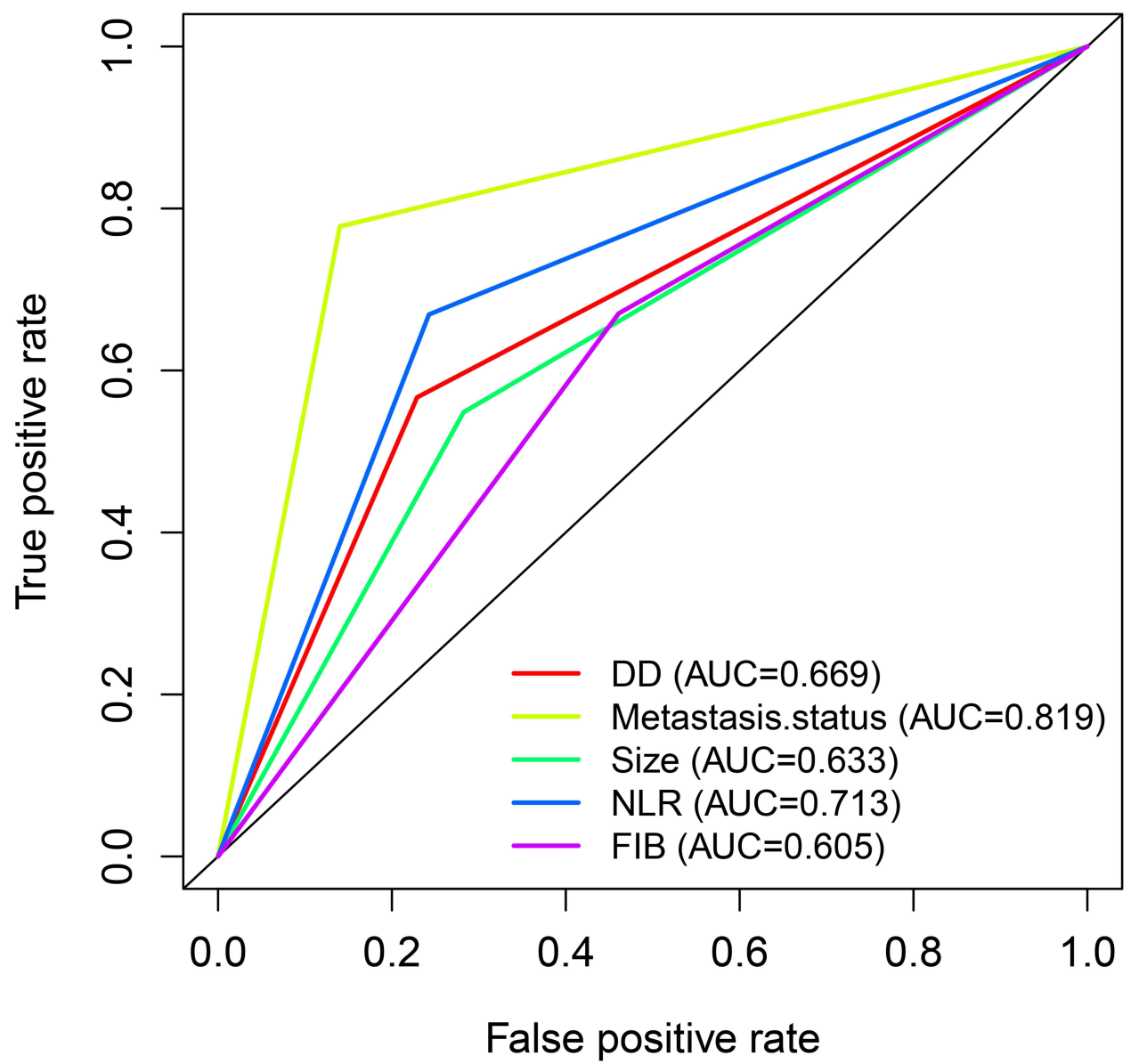

Figure 2 Receiver operating characteristic curve that evaluates the predictive power of variables with significant significance in univariate Cox analysis.

early mortality, among whom early follow-up, such as increasing the frequency of postoperative chest CT and local MRI examinations, should be prioritized.

In our study, tumor metastasis was found to be the main cause of early mortality among people with osteosarcoma. The risk of early mortality in those with localized osteosarcoma was significantly lower than in those with metastatic osteosarcoma. In addition, in our nomogram, tumor metastatic status occupies the most important position. The influence of tumor metastatic status on the prognosis of people with osteosarcoma is well recognized: approximately $20 \%$ of those with osteosarcoma have metastases at diagnosis; in addition, metastasis eventually occurs in $40 \%$ of those with osteosarcoma who do not have metastasis at diagnosis. The current prognosis of metastatic osteosarcoma patients is poor, with a 5 -year OS of only $20-30 \% .^{19,20}$

Our study shows that the preoperative NLR is also associated with early mortality among people with osteosarcoma. Our results show that a high NLR independently predicts a higher risk of early death in this population. In addition, our Kaplan-Meier survival curve analysis demonstrated that people with osteosarcoma who had a low NLR had a better OS than those with a high NLR. In line with our results, other published studies have also demonstrated that a lower preoperative NLR are associated with better prognosis in patients with various cancers, including non-small cell lung cancer and colorectal cancer. ${ }^{21-23}$ Further, preoperative NLR has been included as a prognostic marker in the treatment guidelines for bladder cancer. The specific reasons why a high NLR is related to poor OS in tumor patients are not yet clear. However, previous studies have reported that increased neutrophils are associated with poor prognosis in multiple cancer patients; this increase in neutrophils may provide a microenvironment that promotes tumor occurrence and development through the production of cytokines and chemokines. Neutrophils can also promote metastasis by 
A

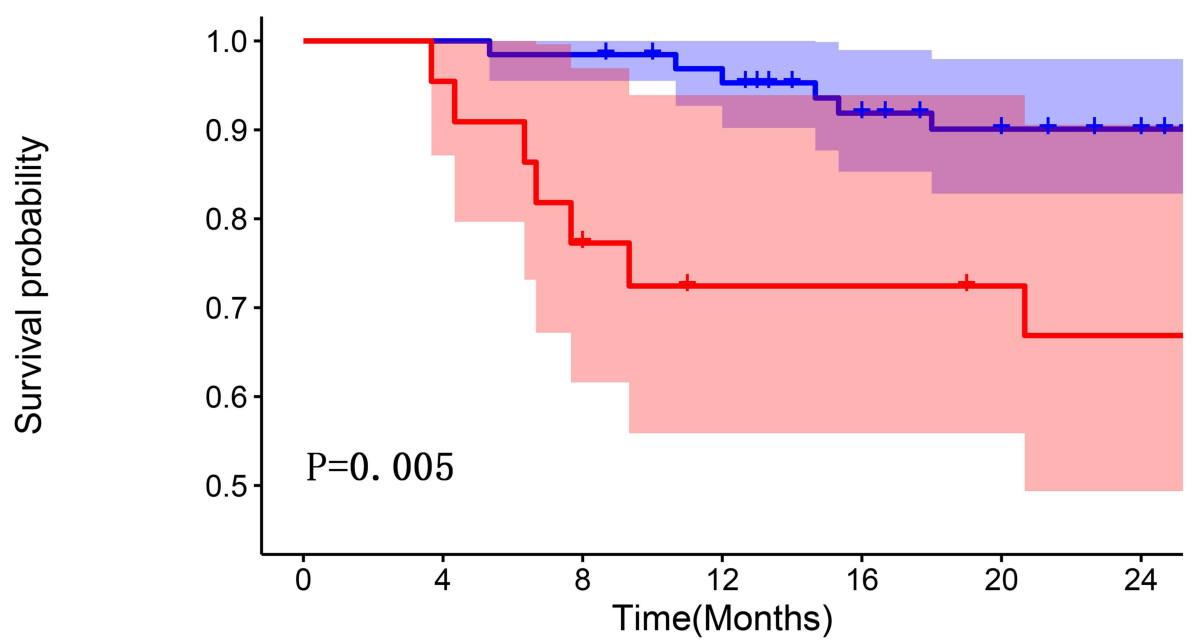

\begin{tabular}{|c|c|c|c|c|c|c|c|}
\hline \multirow{3}{*}{$\begin{array}{l}\frac{\Omega}{N} \mathrm{NLR}<=2.529 . \\
\sum \quad \mathrm{NLR}>2.529 .\end{array}$} & 65 & 65 & 64 & 61 & 54 & 50 & 47 \\
\hline & 22 & 21 & 17 & 14 & 14 & 13 & 12 \\
\hline & 0 & 4 & 8 & $\begin{array}{r}12 \\
\text { (Mc }\end{array}$ & 16 & 20 & 24 \\
\hline
\end{tabular}

B

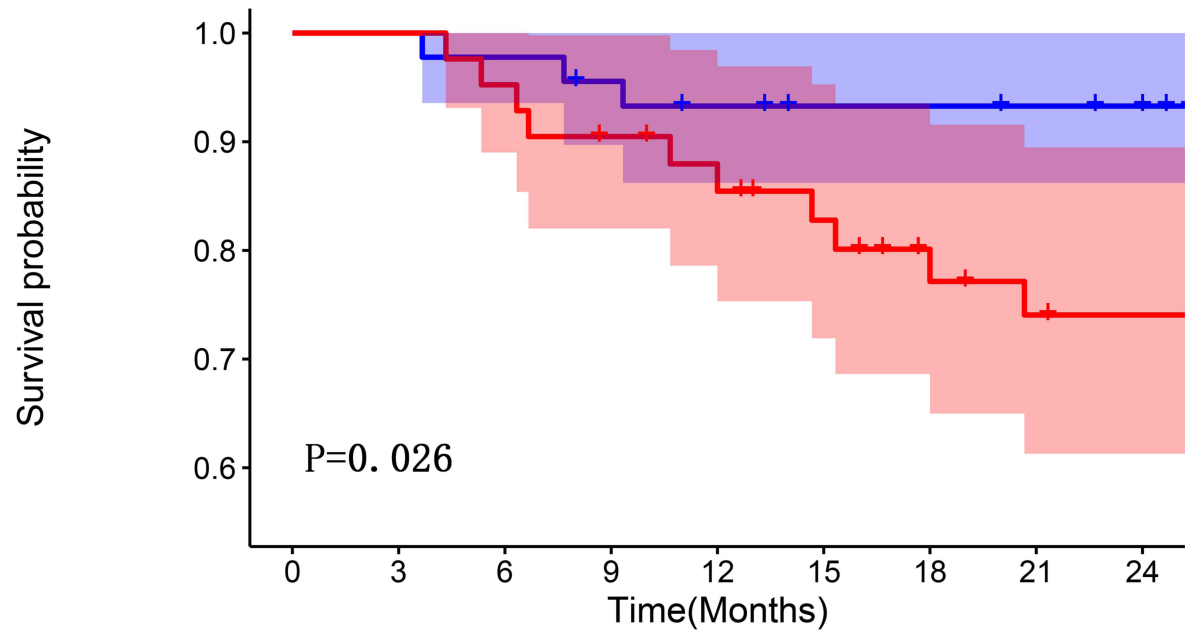

\begin{tabular}{|c|c|c|c|c|c|c|c|c|c|}
\hline \multirow{3}{*}{ 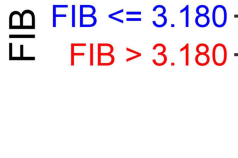 } & 45 & 45 & 44 & 42 & 40 & 38 & 38 & 37 & 36 \\
\hline & 42 & 42 & 40 & 37 & 35 & 31 & 27 & 24 & 23 \\
\hline & 0 & 3 & 6 & 9 & & 15 & 18 & 21 & 24 \\
\hline
\end{tabular}

Figure 3 Evaluation of independent hematological risk factors in predicting early mortality in patients with osteosarcoma. (A) The Kaplan-Meier survival curve indicate that the early overall survival of patients in the low neutrophil-lymphocyte ratio group is better than that of the high neutrophil-lymphocyte ratio group. (B) The Kaplan-Meier survival curve indicate that the early overall survival of patients in the low fibrinogen levels group is better than that of the high fibrinogen levels group. 
A

Points

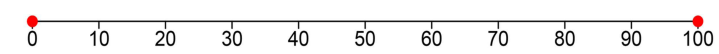

DD

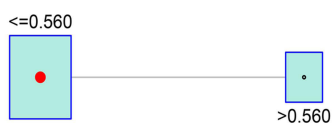

FIB * $^{*}$

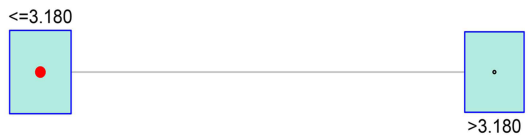

$\mathrm{NLR}^{*}$

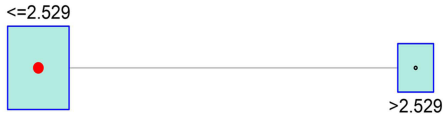

Size

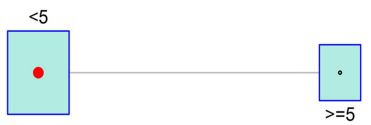

Metastasis.at.diagñsis ${ }^{* * *}$

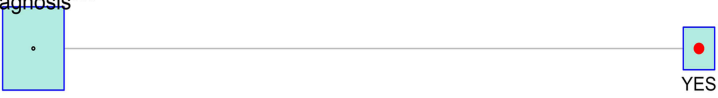

Total points

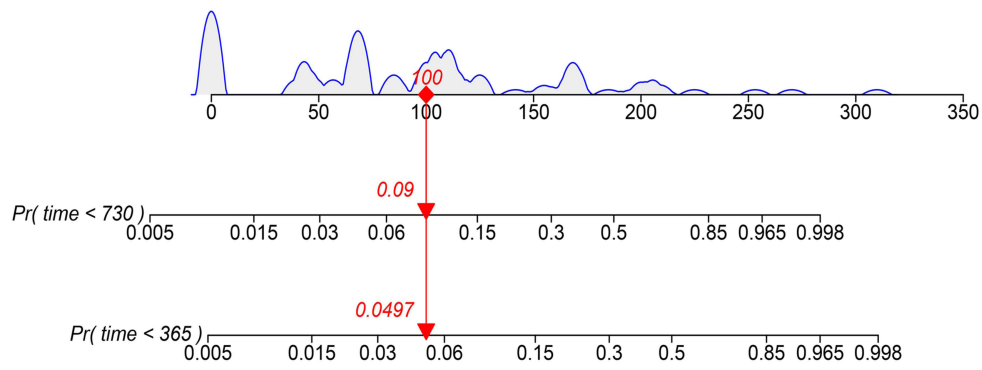

B

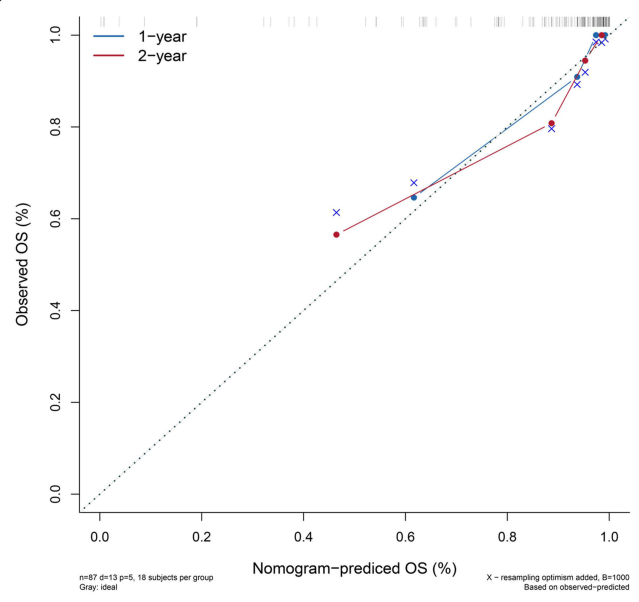

C

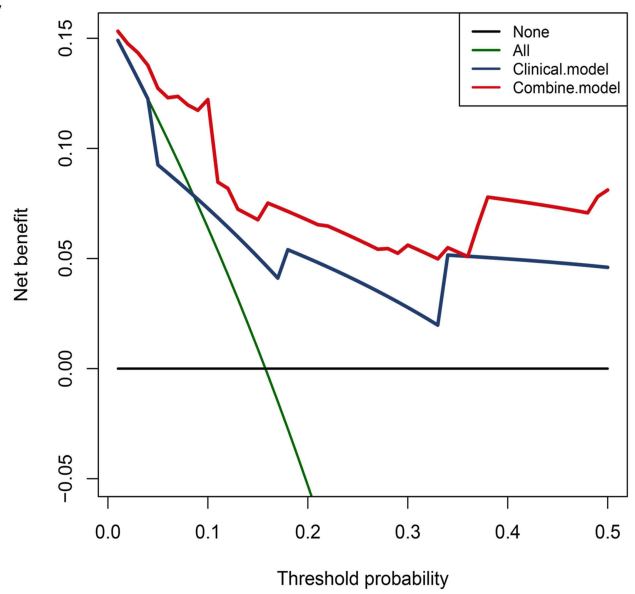

Figure 4 Construct and evaluate the nomogram "Osteosarcoma Early Mortality Nomogram”. (A) "Osteosarcoma Early Mortality Nomogram" constructed based on the significant variables in the univariate Cox analysis. (B) The calibration curve of "Osteosarcoma Early Mortality Nomogram", the calibration curve shows that the model fits the ideal model best when predicting death in I year. (C) Decision curve analysis of "Osteosarcoma Early Mortality Nomogram". *P<0.05, **P<0.0 I, *** $<0.00$ I.

remodeling the extracellular matrix to promote angiogenesis. ${ }^{24}$ Conversely, lymphocytes have a key role in tumor immune surveillance, inhibiting tumor maturation, and neutrophils also inhibit the cytolytic activity of lymphocytes. ${ }^{25,26}$ This may provide a biological mechanism for the observation that high NLR is associated with poor OS.

Finally, we found that FIB level is also an independent prognostic factor in the prediction of early mortality among people with osteosarcoma. Previous studies have shown that coagulation abnormalities occur in approximately $94 \%$ of cancer patients. ${ }^{27,28}$ FIB may reflect the coagulation function of patients, and it is also an acute phase reactive protein, the concentration of which significantly increases when inflammation occurs. ${ }^{29}$ In addition, FIB can also enhance the adhesion of platelets to tumor cells; in turn, platelets release thrombin, which promote the aggregation of FIB. ${ }^{30}$ However, there are few studies on the role of FIB in osteosarcoma, and more research is therefore needed to determine the prognostic value of FIB in osteosarcoma.

This study has some limitations. First, this is a retrospective study, so there may be an inherent selection bias. Secondly, further research is required to determine an optimal cutoff value for NLR and FIB levels in order for this research to be applied in a clinical setting. Thirdly, our cohort did not distinguish between pathological subtypes of patients, which may have resulted in additional bias. Therefore, we suggest that further research should focus on exploring the application of hematological indicators in patients with different subtypes of osteosarcoma. Finally, the 
number of people included in the study was relatively low. Therefore, our results should be interpreted with caution, and well-designed prospective studies are needed to validate our conclusions.

\section{Conclusion}

In short, tumor metastatic status, NLR, and FIB levels are independent risk factors that can predict early mortality in people with osteosarcoma. Our nomogram can be used to identify patients with a high risk of early death, among whom we recommend prioritizing early follow-up of these patients, for example with chest CT or local MRI scans.

\section{Abbreviations}

ALP, alkaline phosphatase; APTT, activated partial thromboplastin time; AUC, area under the receiver operating characteristic curve; CI, confidence interval; CT, computed tomography; DD, D-dimer; FIB, fibrinogen; HR, hazard ratio; KPS, Karnofsky performance status; LMR, lymphocyte-to-monocyte ratio; MRI, magnetic resonance imaging; NLR, neutrophil-to-lymphocyte ratio; OS, overall survival; PLR, platelet-to-lymphocyte ratio; PNI, prognostic nutritional index; PT, prothrombin time; RDW, red blood cell distribution width; ROC, receiver operating characteristic; TROC, time-dependent receiver operating characteristic curves.

\section{Ethics Approval}

The Ethics Committee of the First Affiliated Hospital of Zhengzhou University reviewed and approved the research protocol of this study.

\section{Acknowledgments}

Thanks for the support of the First Affiliated Hospital of Zhengzhou University in all aspects.

\section{Author Contributions}

All authors contributed to data analysis, drafting or revising the article, have agreed on the journal to which the article will be submitted, gave final approval of the version to be published, and agree to be accountable for all aspects of the work.

\section{Funding}

This work was supported by grants from the 2020 Science and Technology Research Project of Henan Provincial Department of Science and Technology (No.202102310214) to T-K.

\section{Disclosure}

The authors report no conflicts of interest in this work.

\section{References}

1. Ritter J, Bielack SS. Osteosarcoma. Ann Oncol. 2010;21 Suppl 7:vii320-325. doi:10.1093/annonc/mdq276

2. Isakoff MS, Bielack SS, Meltzer P, Gorlick R. Osteosarcoma: current treatment and a collaborative pathway to success. J Clin Oncol. 2015;33 (27):3029-3035. doi:10.1200/JCO.2014.59.4895

3. Zhong J, Hu Y, Si L, et al. Clarifying prognostic factors of small cell osteosarcoma: a pooled analysis of 20 cases and the literature. J Bone Oncol. 2020;24:100305. doi:10.1016/j.jbo.2020.100305

4. Roberts RD, Lizardo MM, Reed DR, et al. Provocative questions in osteosarcoma basic and translational biology: a report from the Children's Oncology Group. Cancer. 2019;125(20):3514-3525. doi:10.1002/cncr.32351

5. Ji X, Shan L, Shen P, He M. Circular RNA circ_001621 promotes osteosarcoma cells proliferation and migration by sponging miR-578 and regulating VEGF expression. Cell Death Dis. 2020;11(1):18. doi:10.1038/s41419-019-2204-y

6. Sheng K, Li Y. LncRNA TUG1 promotes the development of osteosarcoma through RUNX2. Exp Ther Med. 2019;18(4):3002-3008. doi:10.3892/ etm.2019.7880

7. Zhou B, Li L, Li Y, Sun H, Zeng C. Long noncoding RNA SNHG12 mediates doxorubicin resistance of osteosarcoma via miR-320a/MCL1 axis. Biomed Pharmacother. 2018;106:850-857. doi:10.1016/j.biopha.2018.07.003

8. Shen H, Deng G, Chen Q, Qian J. The incidence, risk factors and predictive nomograms for early death of lung cancer with synchronous brain metastasis: a retrospective study in the SEER database. BMC Cancer. 2021;21(1):825. doi:10.1186/s12885-021-08490-4 
9. Zhu Y, Fang X, Wang L, Zhang T, Yu D. A predictive nomogram for early death of metastatic gastric cancer: a retrospective study in the SEER database and China. J Cancer. 2020;11(18):5527-5535. doi:10.7150/jca.46563

10. Mahmoud FA, Rivera NI. The role of C-reactive protein as a prognostic indicator in advanced cancer. Curr Oncol Rep. 2002;4(3):250-255. doi:10.1007/s11912-002-0023-1

11. Li LQ, Bai ZH, Zhang LH, et al. Meta-analysis of hematological biomarkers as reliable indicators of soft tissue sarcoma prognosis. Front Oncol. 2020;10:30. doi:10.3389/fonc.2020.00030

12. Liu B, Huang Y, Sun Y, et al. Prognostic value of inflammation-based scores in patients with osteosarcoma. Sci Rep. 2016;6:39862. doi:10.1038/ srep39862

13. Liang YJ, Mei XY, Zeng B, et al. Prognostic role of preoperative D-dimer, fibrinogen and platelet levels in patients with oral squamous cell carcinoma. BMC Cancer. 2021;21(1):122. doi:10.1186/s12885-021-07841-5

14. Wasserman JK, Nicholas G, Yaworski R, et al. Radiological and pathological features associated with IDH1-R132H mutation status and early mortality in newly diagnosed anaplastic astrocytic tumours. PLoS One. 2015;10(4):e0123890. doi:10.1371/journal.pone.0123890

15. Zhu Z, Wang X, Wang J, et al. Preoperative predictors of early death risk in bladder cancer patients treated with robot-assisted radical cystectomy. Cancer Med. 2019;8(7):3447-3452. doi:10.1002/cam4.2237

16. Zhao C, Li L, Guo X, et al. Preoperative predictors of early mortality risk in isocitrate dehydrogenase-wild-type glioblastoma patients treated with standard therapy. Cancer Manag Res. 2021;13:1159-1168. doi:10.2147/CMAR.S290053

17. Karnofsky D, Burchenal J. The clinical evaluation of chemotherapeutic agents in cancer. Eval Chemotherapeutic Agents. 1949;4:191-205.

18. Smeland S, Bielack SS, Whelan J, et al. Survival and prognosis with osteosarcoma: outcomes in more than 2000 patients in the EURAMOS-1 (European and American Osteosarcoma Study) cohort. Eur j Cancer. 2019;109:36-50. doi:10.1016/j.ejca.2018.11.027

19. Bielack SS, Kempf-Bielack B, Delling G, et al. Prognostic factors in high-grade osteosarcoma of the extremities or trunk: an analysis of 1702 patients treated on neoadjuvant cooperative osteosarcoma study group protocols. J Clin Oncol. 2002;20(3):776-790. doi:10.1200/ JCO.2002.20.3.776

20. Kager L, Zoubek A, Pötschger U, et al. Primary metastatic osteosarcoma: presentation and outcome of patients treated on neoadjuvant Cooperative Osteosarcoma Study Group protocols. J Clin Oncol. 2003;21(10):2011-2018. doi:10.1200/JCO.2003.08.132

21. Cupp MA, Cariolou M, Tzoulaki I, Aune D, Evangelou E, Berlanga-Taylor AJ. Neutrophil to lymphocyte ratio and cancer prognosis: an umbrella review of systematic reviews and meta-analyses of observational studies. BMC Med. 2020;18(1):360. doi:10.1186/s12916-020-01817-1

22. Daher S, Lawrence YR, Dudnik E, et al. Nivolumab in non-small cell lung cancer: real world long-term survival results and blood-based efficacy biomarkers. Front Oncol. 2021;11:625668. doi:10.3389/fonc.2021.625668

23. Chen JH, Zhai ET, Yuan YJ, et al. Systemic immune-inflammation index for predicting prognosis of colorectal cancer. World J Gastroenterol. 2017;23(34):6261-6272. doi:10.3748/wjg.v23.i34.6261

24. De Larco JE, Wuertz BR, Furcht LT. The potential role of neutrophils in promoting the metastatic phenotype of tumors releasing interleukin-8. Clin Cancer Res. 2004;10(15):4895-4900. doi:10.1158/1078-0432.CCR-03-0760

25. Kitamura T, Qian BZ, Pollard JW. Immune cell promotion of metastasis. Nat Rev Immunol. 2015;15(2):73-86. doi:10.1038/nri3789

26. Elinav E, Nowarski R, Thaiss CA, Hu B, Jin C, Flavell RA. Inflammation-induced cancer: crosstalk between tumours, immune cells and microorganisms. Nat Rev Cancer. 2013;13(11):759-771. doi:10.1038/nrc3611

27. Lee AY. Cancer and thromboembolic disease: pathogenic mechanisms. Cancer Treat Rev. 2002;28(3):137-140. doi:10.1016/S0305-7372(02)000440

28. Bestari MB, Agustanti N. Obstructive jaundice due to pancreatic metastasis from non-small cell lung cancer. Acta Medica Indonesiana. 2013;45 (3):216-219.

29. Page MJ, Thomson GJA, Nunes JM, et al. Serum amyloid A binds to fibrin (ogen), promoting fibrin amyloid formation. Sci Rep. 2019;9(1):3102. doi:10.1038/s41598-019-39056-X

30. Zheng S, Shen J, Jiao Y, et al. Platelets and fibrinogen facilitate each other in protecting tumor cells from natural killer cytotoxicity. Cancer Sci. 2009;100(5):859-865. doi:10.1111/j.1349-7006.2009.01115.x

Cancer Management and Research

Dovepress

Publish your work in this journal

Cancer Management and Research is an international, peer-reviewed open access journal focusing on cancer research and the optimal use of preventative and integrated treatment interventions to achieve improved outcomes, enhanced survival and quality of life for the cancer patient. The manuscript management system is completely online and includes a very quick and fair peer-review system, which is all easy to use. Visit http://www.dovepress.com/testimonials.php to read real quotes from published authors.

Submit your manuscript here: https://www.dovepress.com/cancer-management-and-research-journal 